\title{
ECMO for immunosuppressed patients with acute respiratory distress syndrome: drawing a line in the sand
}

\author{
Matthieu Schmidt ${ }^{1,2^{*}}$, Alain Combes ${ }^{1,2}$ and Kiran Shekar ${ }^{3,4,5}$
}

(C) 2019 Springer-Verlag GmbH Germany, part of Springer Nature

Acute respiratory failure is a common and frequently fatal condition in immunosuppressed patients [1, 2]. Caring for these intensive care unit (ICU) patients is challenging as clinicians would ideally have to define two major points. The first one is our ability to reasonably prognosticate this evolving heterogeneous group of patients. With advances in oncology, haematology and immunosuppressant treatments [3], intensivists are increasingly encountering immunosuppressed patients with acute respiratory distress syndrome (ARDS), often elderly and frail [4]. The second challenge is defining the upper ceiling of intensive care support, which is frequently based on a favourable prognosis from their primary underlying disease and related immunosuppression. However, in critically ill patients, it is frequently difficult to identify who will benefit from admission to the ICU and lifesustaining interventions when the chances of a successful outcome including good health-related quality of life are unclear. In these circumstances, a time-limited trial of intensive care treatment, aiming to initiate all necessary treatments for a certain period of time to gain a more realistic understanding of the patient's chances of a successful recovery, could be relevant [5]. This strategy may include last-stage adjunct therapies such as extracorporeal membrane oxygenation (ECMO). Should these patients be supported with ECMO? This will remain an important question in times to come as the use of ECMO and immunosuppressant therapies are both on the rise.

*Correspondence: matthieu.schmidt@aphp.fr

${ }^{1}$ Sorbonne Université ", UPMC Univ Paris 06, INSERM UMRS_1166-iCAN, Institute of Cardiometabolism and Nutrition, Paris Cedex 13, France

Full author information is available at the end of the article
The use of ECMO in immunocompromised patients in the context of ARDS is increasing as it concerns 5-31\% of the large ECMO cohorts [6, 7]. Notably, 22\% of the patients in the ECMO to Rescue Lung Injury in Severe ARDS (EOLIA) trial were found to have an immunocompromised condition with $56 \%$ and $78 \% 60$-day mortality in the ECMO and the control groups, respectively [8]. Unsurprisingly, the confrontation of chronically immunocompromised patients with critical illness is associated with a significantly increased mortality risk. A recent retrospective international study in 203 ECMO-supported ARDS immunocompromised patients helps to understand in detail the specific conditions and circumstances that govern the use of ECMO in such highly complicated patient groups [9]. Six-month overall survival was only $30 \%$. However, the pathophysiologic inhomogeneity between cancer patients with solid tumours, patients with haematological malignancies and patients with pharmacologically induced immunosuppression prompts one to carefully identify the subgroup of patients whom are more likely to benefit from this invasive therapy. In this cohort, 6-month survivals were $40 \%, 37 \%, 26 \%, 24 \%$ and $20 \%$ in the solid-organ transplant, long-term or highdose corticosteroids or immunosuppressant, acquired immune deficiency syndrome, haematological malignancies and solid tumour groups, respectively [9]. The significant worse outcomes for patients with haematological malignancies was also highlighted in the context of allogeneic haematopoietic stem cell transplantation where discouraging survival rates (4\% ICU survival) in patients treated early after allogeneic haematopoietic stem cell transplantation do not support the use of ECMO. On the contrary, long-term allogeneic haematopoietic stem cell transplantation recipients otherwise eligible for full-code

\section{Springer}


ICU management may be potential candidates for ECMO therapy in cases of severe ARDS failing conventional measures [10].

Management of ECMO-supported severe ARDS in immunocompromised patients is challenging and further research is warranted to improve their poor outcome. First, low 6-month survival supports restricting ECMO to patients with realistic oncological/therapeutic prognoses, acceptable functional status and few pre-ECMO mortality risk factors. It appears that patients who are unwell enough to receive invasive mechanical ventilation (MV) have similar outcomes to those who then go on to receive ECMO [9]. In this setting, ECMO may be an extension of an intention to support physiology and buy time to reverse underlying pathology. It may come down to an "all or none" approach to invasive respiratory support in immunosuppressed patients with ARDS. Therefore, on the basis of current data eligible patients should receive timely lung-protective invasive MV, adjunct therapies and ECMO as needed if they fulfilled EOLIA inclusion criteria [8]. Less than 30 days between immunodeficiency diagnosis and ECMO cannulation is independently associated with better 6-month survival, which should prompt ICU physicians to apply a full code strategy including ECMO in this population. Second, as expected for this at-risk population, ECMOrelated bleeding and nosocomial infections are frequent $[9,10]$. ECMO-related major haemorrhages, ventilatorassociated pneumonias and cannula infections occur in $36 \%, 50 \%$ and $10 \%$ of the patients, respectively, and lower pre-ECMO platelet count is significantly associated with 6-month mortality [9]. Because outcomes on ECMO are comparable to outcomes with invasive MV in that population, even though most patients receive ECMO after failing invasive $M V$, it might be a valuable option to block the vicious cycle that negatively affects these patients on MV. However, it is unclear if these outcomes are mostly related to primary underlying conditions, immunosuppression and disease severity or additional risks of invasive MV and/or ECMO (Table 1).
In the cohort reported by Schmidt et al., the non-survivors, apart from being immunosuppressed, also demonstrated higher driving pressures and higher $\mathrm{PaCO}_{2}$ levels before cannulation, which was consistent with previous ECMO studies [6, 7]. Third, avoiding invasive MV is an objective in that population, as it strongly impacts the outcome [11]. In that context, VV-ECMO might be an interesting alternative. With this rationale, a singlecentre, uncontrolled, pilot trial assessed VV-ECMO feasibility in awake, non-intubated, spontaneously breathing ARDS patients enrolled in six patients (four immunocompromised) [12]. Three of the four hospital survivors were successfully managed without invasive MV after 10 , 5 and 7 days on ECMO. Notably, if ECMO-related bleeding could be prevented, ECMO for awake, non-intubated, spontaneously breathing ARDS patients might offer major theoretical advantages: prevention of prolonged invasive MV complications (i.e. ventilator-associated pneumonia, ventilator-induced lung injuries etc.), maintenance of physical activity, social interaction, oral feeding, spontaneous coughing and low sedation requirement [13]. The control of the patient's respiratory drive and the application of an intermittent optimized positive endexpiratory pressure, through a non-invasive interface (high flow oxygen or non-invasive ventilation) to prevent lung derecruitment, are the key factors to making this strategy successful. However, this intriguing approach, still experimental to date, warrants further supporting data, as spontaneous breathing could provide itself significant lung damage [14] and controversial results have been reported so far [10]. To date, while awaiting more data to support new approaches [15], clinicians should be aware that immunocompromised patients who are unlikely to benefit from invasive MV are unlikely to benefit from ECMO and should therefore receive comfort-oriented care if non-invasive treatment options fail. Fourth, the high burden of ECMO raises numerous ethical questions. Patients and families need to clearly understand that clinicians may embark on a resource-intense, highrisk, invasive therapy with a smaller chance of survival

\section{Table 1 Open research questions in the context of ECMO-supported severe ARDS in immunocompromised patients}

\begin{tabular}{ll}
\hline Indications & Which sub-population of haematological patients may likely benefit from ECMO? \\
Management & Are the pre-ECMO prognosis risk models suitable to predict outcome in that population? \\
& How to decrease the burden of ECMO-related bleeding complication? \\
& Which strategies to reduce the high risk of infectious complication? Should we use prophylactic antibiotics? \\
& Should we propose ECMO before invasive mechanical ventilation? \\
& Is awake ECMO a safe and beneficial option? \\
Ethics & How to integrate all stakeholders, including the patient and their family, to share decision-making? \\
& How and when to identify the time where full code trial of intensive care treatment switch to futility?
\end{tabular}

ARDS acute respiratory distress syndrome, ECMO extracorporeal membrane oxygenation 
and no guarantee of a functional outcome in this immunocompromised population. In that context, benefits and limitations of ECMO prior to its initiation should be clearly explained to the patient and/or their surrogates. Later on, physician decisions regarding continuation or withdrawal of ECMO have to be done according to patient's or surrogate's wishes, and perceived "futility" of the clinical situation. Given the complexity of such situations, shared decision-making that includes all stakeholders, including the patient's family, is crucial.

In summary, ICUs are likely to admit more immunosuppressed patients in future [4]. The existing literature provides useful insights into patient populations that may benefit from ECMO. Patients with solid organ transplants and shorter duration of immunosuppression ( $<30$ days) appear to have a more favourable survival rate [9]. Awake ECMO might be an attractive strategy which, however, warrants further investigations. Meanwhile, early ICU admission and early identification of the underlying cause of respiratory failure may assist with early risk stratification and prognostication.

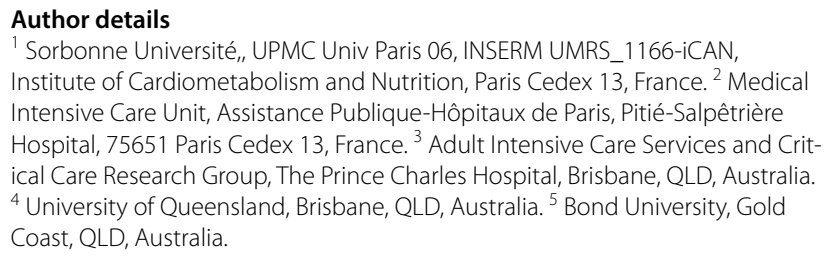

Compliance with ethical standards

\section{Conflicts of interest}

Matthieu Schmidt has received lectures fees from Getinge, Xenios and Dräger. Alain Combes has received lecture fees from Baxter and Getinge. Kiran Shekar has no conflicts of interest.

\section{Publisher's Note}

Springer Nature remains neutral with regard to jurisdictional claims in published maps and institutional affiliations.

Received: 18 March 2019 Accepted: 25 April 2019

Published online: 13 May 2019
References

1. Canet E, Zafrani L, Azoulay É (2016) The critically ill kidney transplant recipient: a narrative review. Chest 149:1546-1555

2. Azoulay E, Pène F, Darmon M et al (2015) Managing critically ill hematology patients: time to think differently. Blood Rev 29:359-367

3. Lueck C, Stadler M, Koenecke C et al (2018) Improved short- and long-term outcome of allogeneic stem cell recipients admitted to the intensive care unit: a retrospective longitudinal analysis of 942 patients. Intensive Care Med 44:1483-1492

4. Harpaz R, Dahl RM, Dooling KL (2016) Prevalence of immunosuppression among US adults, 2013. JAMA 316:2547-2548

5. Vink EE, Azoulay E, Caplan A et al (2018) Time-limited trial of intensive care treatment: an overview of current literature. Intensive Care Med 44:1369-1377

6. Schmidt M, Zogheib E, Roze H et al (2013) The PRESERVE mortality risk score and analysis of long-term outcomes after extracorporeal membrane oxygenation for severe acute respiratory distress syndrome. Intensive Care Med 39:1704-1713

7. Schmidt M, Bailey M, Sheldrake J et al (2014) Predicting survival after extracorporeal membrane oxygenation for severe acute respiratory failure. The Respiratory Extracorporeal Membrane Oxygenation Survival Prediction (RESP) score. Am J Respir Crit Care Med 189:1374-1382

8. Combes A, Hajage D, Capellier G et al (2018) Extracorporeal membrane oxygenation for severe acute respiratory distress syndrome. N Engl J Med 378:1965-1975

9. Schmidt M, Schellongowski P, Patroniti N et al (2018) Six-month outcome of immunocompromised severe ARDS patients rescued by ECMO. An international multicenter retrospective study. Am J Respir Crit Care Med

10. Wohlfarth P, Beutel G, Lebiedz P et al (2017) Characteristics and outcome of patients after allogeneic hematopoietic stem cell transplantation treated with extracorporeal membrane oxygenation for acute respiratory distress syndrome. Crit Care Med 45:e500-e507

11. Azoulay E, Mokart D, Pene F et al (2013) Outcomes of critically ill patients with hematologic malignancies: prospective multicenter data from France and Belgium - a groupe de recherche respiratoire en reanimation onco-hematologique study. J Clin Oncol 31:2810-2818

12. Hoeper MM, Wiesner O, Hadem J et al (2013) Extracorporeal membrane oxygenation instead of invasive mechanical ventilation in patients with acute respiratory distress syndrome. Intensive Care Med 39:2056-2057

13. Langer T, Vecchi V, Belenkiy SM et al (2014) Extracorporeal gas exchange and spontaneous breathing for the treatment of acute respiratory distress syndrome: an alternative to mechanical ventilation? Crit Care Med 42:e211-e220

14. Yoshida T, Fujino Y, Amato MBP, Kavanagh BP (2017) Fifty years of research in ARDS. Spontaneous breathing during mechanical ventilation. risks, mechanisms, and management. Am J Respir Crit Care Med 195:985-992

15. Combes A, Brodie D, Chen Y-S et al (2017) The ICM research agenda on extracorporeal life support. Intensive Care Med 43:1306-1318 